\title{
ARTIGO
}

dO https://doi.org/10.22481/praxisedu.v15i34.5637

\section{PESQUISA QUANTITATIVA E EDUCAÇÃO: DESAFIOS E POTENCIALIDADES}

\author{
QUANTITATIVE RESEARCH AND EDUCATION: CHALLENGES AND \\ POTENTIALITIES
}

\author{
INVESTIGACIÓN CUANTITATIVA Y EDUCACIÓN: DESAFÍOS Y \\ POTENCIALIDADES
}

\author{
Graciela da Silva Oliveira \\ Universidade Federal de Mato Grosso - Brasil \\ Ana Paula Zampieri Silva de Pietri \\ Associação Cidade Escola Aprendiz - Brasil
}

\author{
Nelio Bizzo \\ Universidade de São Paulo- Brasil \\ Universidade Federal de São Paulo - Brasil
}

\begin{abstract}
Resumo: A realização de pesquisas em larga escala na área da educação requer acesso a uma grande produção de dados, que envolve a participação de muitos sujeitos colaboradores. Considerando-se as dimensões geográficas do Brasil, com suas particularidades regionais, é imprescindível uma logística eficiente para a execução exitosa do estudo. Pensando nisso, o presente trabalho tem como objetivo apresentar algumas reflexões sobre as experiências com pesquisas de cunho quantitativo e de larga escala no âmbito do ensino de ciências, caracterizando aspectos teórico-metodológicos, alguns desafios e as possibilidades de investigação ao se obter dados de representatividade nacional. Os resultados apontam que, ao delinear uma pesquisa com essas características, o trabalho colaborativo deve se fazer presente, a fim de possibilitar a integração e discussão entre todos os sujeitos envolvidos na pesquisa. Além disso, é preciso considerar as especificidades das escolas e da universidade, de modo que ambas as instituições dialoguem e ocupem o lugar de formadoras durante o processo de obtenção e tratamento dos dados da investigação.
\end{abstract}

Palavras chave: Amostra nacional; Quantitativo; Questionários.

\begin{abstract}
Developing large-scale research in education requires access to a great amount of data, which involves the participation of many collaborators. In addition to considering Brazilian geographical dimension and its regional particularities, it is necessary to have an efficient logistics for the successful development of studies. This paper shares and reflects on a number of quantitative and large-scale research in the context of science teaching pointing out theoretical-methodological aspects, some challenges and the investigation possibilities to obtain nationwide representative data. The results demonstrate that collaborative work should be part of research providing the integration and discussion among all those involved in the research. When designing research with these
\end{abstract}


characteristics it is necessary to consider the particularities of the schools and universities so that all institutions interact and perform as trainers during data generation and treatment.

Keywords: National sample; Quantitative; Questionnaires.

Resumen: La realización de investigaciones a gran escala en el área de la educación requiere el acceso a una gran producción de datos. También, implica la participación de muchos colaboradores y, además, teniendo en cuenta las dimensiones geográficas de Brasil, con sus particularidades regionales, exige una logística eficiente para la implementación exitosa del estudio. El presente trabajo tiene por objetivo presentar algunas reflexiones sobre las experiencias con la investigación cuantitativa y de gran escala en el campo de la enseñanza de ciencias, caracterizando aspectos teórico-metodológicos, algunos retos y posibilidades de investigación cuando se obtienen datos de representatividad nacional. Los resultados indican que es necesario el trabajo colaborativo, pues permite la integración y el debate entre todos los sujetos involucrados en la investigación. Al delinearse una investigación con esas características, es necesario tener en cuenta las especificidades de las escuelas y de las universidades para que ambas instituciones dialoguen y ocupen el lugar de formadores durante el proceso de obtención y tratamiento de los datos de Investigación.

Palabras-clave: Muestra nacional; Cuantitativo; Cuestionarios.

\section{Introdução}

Na pesquisa em educação, de acordo com Gatti (2004), exceto pelas avaliações de desempenho escolar, poucos estudos são conduzidos com o emprego de técnicas e métodos quantitativos; pode-se afirmar que há pouca tradição ou utilização desse método de análise e tratamento dos dados. A autora destaca que "há mais de duas décadas que na formação de educadores e de mestres e doutores em educação não se contemplam estudos disciplinares sobre esses métodos" (GATTI, 2004, p. 13). Tal situação parece persistir no cenário atual, uma vez que, no meio educacional, ainda há maior número de estudos de cunho qualitativo.

Indiscutivelmente as abordagens qualitativas nas pesquisas têm enriquecido a compreensão de diversos aspectos educacionais, visto que são priorizados estudos dos objetos e situações-chave sob a ótica da interpretação dos sujeitos sociais, com atenção às nuances do particular. No entanto, para Gatti (2004, p. 13), existem aspectos, objetos e situações do campo educacional que "para sua contextualização e compreensão necessitam ser qualificados através de dados quantitativos". A autora exemplifica o caso com trabalhos que versam sobre analfabetismo, percurso e fracasso escolar; fluxo escolar; letramento; políticas públicas e financiamento de educação básica; avaliação educacional, entre outros.

Os estudos de abordagem quantitativa investigam situações sociais, baseando-se no entendimento aprofundado de variáveis, medidas e suas relações numericamente analisadas 
mediante os procedimentos estatísticos (CRESWELL, 1994). Por vezes, essa interpretação contribui para uma percepção do paradigma quantitativo como uma análise dos fatos dissociados da realidade do sujeito. Neste caso, os pesquisadores estariam mais preocupados com as tendências numéricas universais em detrimento da compreensão da particularização. Entretanto, as abordagens quantitativas podem ajudar a: contribuir com a compreensão das opiniões e concepções de grandes amostras representativas de comunidades culturais; confirmar ou refutar hipóteses teóricas; e resumir dados numéricos de maneira clara para as políticas públicas (FASSINGER; MORROW, 2013).

De naturezas distintas, as bases pragmáticas de abordagens quantitativas e qualitativas podem diferir suficientemente para serem interpretadas como incompatíveis. No entanto, Minayo e Sanches (1993) argumentam que, do ponto de vista metodológico, não são contraditórias.

A abordagem quantitativa atua em níveis da realidade, em que os dados se apresentam aos sentidos, e tem como prática trazer à luz dados, indicadores e tendências, classificando-os e tornando-os inteligíveis por meio de análise de variáveis. Já a abordagem qualitativa trabalha com valores, crenças, representações, opiniões e hábitos analisados com maior profundidade a partir de fenômenos, fatos e processos particulares e específicos (MINAYO; SANCHES, 1993). Para os autores, não se trata de apresentar uma abordagem em oposição à outra, mas cabe desenvolver as pesquisas no campo educacional e social a partir da associação de diversos métodos e técnicas, de modo a analisar os diferentes aspectos e seus significados, com o quantitativo gerando questões para serem aprofundadas qualitativamente e vice-versa.

Para Flick (2009), as discussões metodológicas que permearam durante muito tempo entre os pesquisadores dos diferentes campos de conhecimento sobre a pesquisa qualitativa e a quantitativa eram de que ambas deveriam ser reconhecidas como diferentes em termos teóricos, epistemológicos e de pesquisa. Essa argumentação distintiva contribuiu para o avanço e o aprimoramento interno dos dois métodos, porém esse desenvolvimento aconteceu paralelamente e de modo independente. Flick (2009) destaca que atualmente essa separação estrita tem sido superada a partir da noção de que os dois métodos devem ser considerados como campos complementares e não díspares e que a combinação de ambos contribui para a qualidade da pesquisa.

Conhecer adequadamente as características, contribuições e limitações de cada abordagem metodológica é condição fundamental para que o pesquisador conduza os seus estudos conforme as peculiaridades de cada uma, no seu devido contexto, conforme os 
objetivos e problemas da pesquisa, e de acordo com a disponibilidade de tempo e recursos disponíveis (SAMPIERI; COLLADO; LUCIO, 2006).

Considera-se, portanto, que a explicitação das possíveis relações entre ambas favorece o entendimento da questão. Não se pretende, contudo, apresentar uma discussão aprofundada sobre os paradigmas que vêm sustentando ambas as perspectivas metodológicas. O intuito é apresentar algumas fundamentações teórico-metodológicas que conduziram as escolhas dos métodos e técnicas de cunho quantitativo empregados em duas pesquisas que buscaram investigar a percepção pública dos jovens brasileiros sobre a ciência.

Além de relatar tais experiências de pesquisas de cunho quantitativo na área de ensino de ciências, caracterizando aspectos teóricos e metodológicos, este artigo busca apresentar seus desafios e possibilidades de execução exitosa.

As referidas pesquisas são decorrentes de estudos quantitativos realizados em colaboração com os projetos Relevance of Science Education (ROSE) e Saberes do Alunado na Perspectiva Internacional: Evolução, Natureza, e Sociedade (SAPIENS), cujo objetivo era investigar opiniões de estudantes na faixa etária de 15 anos de idade sobre suas experiências nas aulas de ciências, opiniões e atitudes sobre ciência e tecnologia, questões ambientais, e conhecimento e aceitação da teoria da evolução biológica.

\section{Desenho das pesquisas ROSE e SAPIENS}

Nos projetos ROSE e SAPIENS, realizados no Brasil entre os anos de 2010 a 2015, foram obtidas amostras de representatividade nacional de estudantes matriculados no Ensino Médio em escolas públicas e privadas brasileiras. Os estudantes colaboradores responderam a um questionário com questões que versavam sobre ciências, tecnologia e os seus desdobramentos. Por serem pesquisas de larga escala, que requerem a participação de muitos sujeitos como colaboradores, deparou-se com algumas dificuldades inerentes às características e peculiaridades da metodologia quantitativa. Entre essas dificuldades, ressaltam-se o procedimento de envio dos questionários e o retorno de um grande percentual, preservando a integridade dos dados a serem analisados.

As escolas analisadas nas pesquisas que integraram os projetos ROSE e SAPIENS foram sorteadas através do processo de amostragem estratificada por conglomerados em dois estágios: escola e turma. Por estarem localizadas em regiões marcadamente díspares, a visita dos pesquisadores responsáveis a todas as instituições para a aplicação do questionário foi 
inviabilizada. Desse modo, para garantir o envio e a devolução dos questionários pelo correio, tornou-se imprescindível a colaboração tanto dos professores quanto dos gestores das escolas participantes. No entanto, alguns fatores interferiram na proporção de respostas, como será visto a seguir.

Após o planejamento amostral e a seleção da população-alvo, iniciou-se o desenvolvimento da logística para aplicação dos questionários em diversas localidades brasileiras e adotaram-se os seguintes critérios: 1) composição de uma equipe de pesquisa responsável pela coleta de dados; 2) contatos com as escolas e solicitação de professores colaboradores; 3) envio de orientações e questionários impressos por correio; 4) aplicação e devolução dos questionários, pelos professores colaboradores, sob a orientação da equipe de pesquisa.

A opção por obter os dados para a realização das pesquisas integradas aos projetos ROSE e SAPIENS a partir de respostas aos questionários enviados por correio foi amparada por algumas experiências e discussões anteriores publicadas na literatura. De acordo com Gouveia e Günther (1995), as vantagens desse método são a redução de gastos e tempo nas coletas, bem como a obtenção de um número maior de sujeitos contatados ao mesmo tempo. Por outro lado, a desvantagem é o baixo percentual de resposta, o que, consequentemente, contribui para problemas de generalização dos resultados obtidos de amostras incompletas. Os questionários enviados para os sujeitos pelo correio, geralmente, apresentam uma proporção de devolução de aproximadamente 25\% (MARCONI; LAKATOS, 2007), o que inviabilizaria a realização das pesquisas as quais reportamos, que estimavam um retorno de aproximadamente $80 \%$ dos questionários respondidos. Importante, destacar que altas taxas de recusas ou perdas inviabilizam as pesquisas quantitativas, de maneira, que o estabelecimento do quantitativo amostral mínimo deverá ser ajustado, considerando o índice de recusas e perdas, evitando estimativas amostrais incompletas.

De acordo com Helgeson, Voss e Terpening (2002), alguns fatores são mais eficazes para aumentar as respostas de instrumentos de pesquisa enviados por correio ou via internet, como os incentivos e as benfeitorias oferecidas aos sujeitos, assim como as atitudes e concepções sobre pesquisa dos respondentes e os demais sujeitos envolvidos com o estudo. Os autores consideram que o número de respostas está ligado a um processo de persuasão, assim, o papel dos pesquisadores é criar uma estratégia de comunicação persuasiva, de modo que o respondente atenda ao desejado. 
Durante a comunicação com o sujeito das diferentes modalidades de pesquisa, dois fatores devem emergir das negociações: a atenção e a intenção de responder aos instrumentos apresentados pelos pesquisadores. Nas pesquisas realizadas através de correio ou internet, ainda há o esforço adicional para os sujeitos de devolverem os instrumentos de coleta de dados preenchidos.

No que se refere a essa questão, os esforços de atenção, intenção e o retorno dos questionários respondidos dependiam diretamente dos gestores e dos professores das escolas envolvidos com a pesquisa. A participação dos vários segmentos da escola (alunos, professores e gestores) foi fundamental para o desenvolvimento das pesquisas, além de todos os sujeitos que ocuparam o lugar de formadores, uma vez que as intervenções de todos os grupos acerca das metodologias de análise foram consideradas pelos pesquisadores responsáveis.

Assim, de modo a minimizar o problema do baixo percentual de respostas, foi realizado, pelo grupo de pesquisadores envolvidos com as pesquisas integrantes dos projetos ROSE e SAPIENS, contato telefônico com os responsáveis (diretores ou coordenadores) das escolas selecionadas no estudo amostral, a fim de verificar a possibilidade de participação na pesquisa e esclarecer aspectos da aplicação do instrumento.

Para reduzir os problemas com o retorno das escolas, ainda foram considerados alguns aspectos levantados por Gouveia e Günther (1995) que parecem influenciar na devolução dos questionários respondidos por correio. Os autores consideram que as características do envelope têm importância no efeito de retorno de questionários respondidos e ressaltam que são poucos os estudos que levam isso em consideração.

De acordo com Helgeson, Voss e Terpening (2002), quando se considera o processo de pesquisa-resposta, a atenção atribuída à pesquisa inicia-se no primeiro contato com o envelope e o seu conteúdo, assim, as características do envelope, como a presença de um carimbo institucional, podem causar impacto positivo. Quanto ao conteúdo, uma carta de apresentação, com as características do instrumento de coleta de dados, deve atrair mais a atenção dos sujeitos colaboradores.

Nesse sentido, nas pesquisas integrantes aos projetos ROSE e SAPIENS, a variável envelope foi contemplada, no sentido de que cada um apresentava o carimbo institucional da Universidade de São Paulo (USP), garantindo que se tratava de um estudo desenvolvido por um grupo de pesquisa vinculado a uma instituição de ensino. Além disso, conforme Gouveia e Günther (1995), um carimbo ou selo de retorno no envelope favorece uma proporção de 
resposta superior. Isso foi constatado, uma vez que o envio de um envelope selado, que deveria ser utilizado para postagem de devolução, favoreceu o retorno dos questionários, pois a escola não teria nenhum custo adicional com o correio.

O segundo conjunto de variáveis apresentado por Gouveia e Günther (1995) foi acerca do formato e acabamento do questionário. No questionário aplicado, houve a preocupação com a redução dos itens apresentados e com o layout final do documento, reestruturado no formato de um caderno de itens, evitando-se o uso de questões e seções com o layout repetitivo. Por último, Gouveia e Günther (1995) ressaltam que alguns incentivos são importantes e contribuem para aumentar as chances de maior resposta dos questionários respondidos, como uma boa apresentação dos objetivos da pesquisa pelos investigadores, o frequente contato pessoal, via correio ou telefone, e retornos materiais e benfeitorias para os sujeitos.

Nesse sentido foram previstas as seguintes ações, que pareceram contribuir com o retorno dos questionários respondidos nas escolas participantes: contatos via telefone e e-mail com os professores colaboradores ao longo da pesquisa a fim de sanar dúvidas e garantir que os prazos para aplicação e devolução fossem atendidos; envio de um livro com a apresentação dos resultados anteriores da equipe de pesquisa; convite ao professor que aplicou os questionários para um encontro a fim de trocar experiências com os demais docentes do país que participaram do projeto, e também para participar de um evento acadêmico, "Encontro de História e Filosofia da Biologia". Por fim, cada escola recebeu um retorno com os resultados das pesquisas, por meio de gráficos, os quais comparavam os dados da escola-sede do professor colaborador com os dados nacionais.

Após o sorteio das escolas que integraram os projetos ROSE e SAPIENS, a equipe composta por pesquisadores da Faculdade de Educação da USP confirmou os números de telefones e os endereços de cada uma das instituições através de acesso ao site do Ministério de Educação (http://www.mec.gov.br/). Os contatos por telefone passaram, então, a ser realizados sistematicamente. Foi elaborado um discurso comum, reproduzido por todos os pesquisadores durante o contato com as escolas participantes, visando apresentar detalhadamente as metodologias e os objetivos das pesquisas que seriam realizadas colaborativamente entre as escolas e a Universidade. Durante o desenvolvimento do SAPIENS-Brasil, os contatos telefônicos aconteceram entre os meses de março a maio de 2014, com as 100 escolas sorteadas; contudo, nos contatos iniciais, algumas se recusaram a 
participar e, por isso, utilizou-se uma lista reserva de escolas sorteadas $(\mathrm{N}=20)$, a fim de garantir o envio dos questionários a 100 escolas, como estipulado no plano amostral.

Após a confirmação de interesse em participar da pesquisa, os questionários para preenchimento foram postados, via correio, acompanhados dos seguintes documentos: Carta de Apresentação do Projeto de Pesquisa; Ficha com as Instruções de Aplicação e Devolução dos Questionários; Termo de Consentimento Livre e Esclarecido (que deveria ser assinado por um responsável da escola); Formulário para Registros/Opiniões sobre a Coleta de Dados e Questionário; e um exemplar do livro Jovens e a Ciência, com resultados de trabalhos anteriores da equipe e pesquisadores do projeto ROSE.

Os questionários foram impressos em folhas ópticas, com personalização a laser das folhas de respostas. Esse método minimizou o tempo e os erros de digitação oriundos da tabulação manual dos dados, além de identificar cada questionário com um código de barras, que, mesmo anônimo, pode ser localizado pelo seu código correspondente. O primeiro teste da aplicação de questionário com uso dessa tecnologia foi realizado e descrito por SantosGouw (2013) e Mota (2013).

Cada escola contatada indicou um professor, responsável pela aplicação e devolução dos questionários. Por se tratar de projeto de pesquisa relacionado aos temas de Ciências e de Biologia, os professores indicados atuavam com o ensino de ambas as disciplinas. Solicitouse ao professor colaborador que retornasse os questionários no máximo em três meses após a sua aplicação e que preenchesse um formulário de descrição da coleta de dados, bem como fornecesse opiniões e comentários sobre o questionário elaborado. Assim, foram observadas e registradas informações sobre: composição da turma que participou da pesquisa; local e horário da aplicação; observações sobre as reações dos jovens enquanto respondiam ao questionário, por exemplo, manifestações de cansaço, interesse, motivação, dúvidas e comentários; e tempo utilizado para o preenchimento. Além disso, o professor podia descrever a forma de exposição dos objetivos e orientações do preenchimento do questionário, apontar principais dúvidas e dificuldades da aplicação e inserir, quando desejado, opiniões e comentários sobre o questionário. Essas informações foram consideradas para o aprimoramento do instrumento a ser aplicado em pesquisas futuras. Todos os professores que aplicaram os questionários devolveram o formulário preenchido.

Por meio das informações descritas pelos professores colaboradores, constatou-se que os questionários foram preenchidos no espaço da sala de aula entre 30 a 50 minutos, 
considerando a apresentação dos objetivos da pesquisa, esclarecimentos sobre preenchimento, distribuição e obtenção das respostas dos estudantes.

No que se refere à proporção de resposta e negociação entre pesquisadores e colaboradores das escolas, alguns aspectos relacionados às recusas em participar da pesquisa por parte das instituições e que também pareceram influenciar as escolas que não devolveram os questionários preenchidos via correio merecem especial atenção.

Algumas características das relações entre universidade e a escola, bem como atitudes e percepções dos envolvidos sobre a pesquisa científica, pareceram influenciar a proporção de respostas obtidas em todas as pesquisas integradas aos projetos ROSE e SAPIENS. Essas inferências corroboram as ideias de Helgeson, Voss e Terpening (2002), as quais destacam que as atitudes positivas com relação à pesquisa científica parecem ser determinantes para intenção e participação do sujeito. Além disso, os autores comentam que experiências anteriores com pesquisas devem influenciar a participação dos sujeitos em investigações futuras. Desta forma, além dos cuidados previstos e executados para favorecer a intenção e participação dos gestores e professores das escolas na pesquisa, o encontro e a parceria entre a universidade e a escola mostraram-se como desafios.

\section{Pesquisa educacional: o encontro da universidade e a escola}

A colaboração entre universidade e escola pode ser definida como um processo interativo entre indivíduos e organizações, com experiências e recursos diversos, que se unem em favor de objetivos comuns, bem como para gerar soluções para problemas complexos. Desse modo, as relações colaborativas entre universidade e escola seriam mutuamente benéficas (MILLER; HAFNER, 2008).

Assim, como parte da proposta inicial dos estudos aqui relatados, houve a preocupação entre os pesquisadores de proporcionar um maior envolvimento dos professores da escola básica com as pesquisas e os seus resultados. Entretanto, os primeiros contatos com a gestão de algumas escolas brasileiras evidenciaram uma tensão na relação entre universidade e a escola.

$\mathrm{Na}$ parceria universidade e escola, geralmente, há diferentes entendimentos de investigação, de seus objetivos e seu papel para o ensino. Entre esses entendimentos ainda há as concepções sobre o trabalho dos colegas da universidade, o que é ser um professor e como os papéis dentro da parceria devem funcionar. Diante dos múltiplos significados que 
permeiam as relações entre a universidade e a escola, surgem, portanto, tensões e conflitos que precisam ser compreendidos e discutidos a fim de que as parcerias sejam valiosas para as duas partes (MCLAUGHLIN; BLACK-HAWKINS, 2004).

No contexto brasileiro, de acordo com Telles (2002), quando os pesquisadores acadêmicos chegam à escola para o desenvolvimento de parcerias e pesquisas geralmente não encontram receptividade e aceitação imediatas pelos gestores e professores das escolas. Telles (2002) comenta que existem múltiplas razões para tal segregacionismo, que fomenta o abismo entre a universidade e a escola. A principal razão refere-se ao modo como os acadêmicos desenvolvem suas pesquisas, pois, ao buscar parceria com a instituição, geralmente utilizam a escola como um campo de coleta de dados: os pesquisadores entram nas salas de aulas, recolhem discursos, fazem registros de cada situação e contexto que o campo educacional disponibiliza, e, ao final da pesquisa, não retornam às escolas para partilharem os resultados do estudo com aqueles que contribuíram para o seu desenvolvimento (TELLES, 2002).

Esse histórico pareceu evidente quando os pesquisadores dos projetos aqui reportados, realizados no âmbito nacional, depararam-se com a recusa por parte de alguns gestores já no primeiro contato. Foram várias as razões para justificá-la, como: falta de tempo para se envolver com projetos de pesquisas; excesso de atribuições escolares; falta de professores interessados; envolvimento com pesquisas anteriores que não resultaram em contribuições efetivas para a escola ou que não cumpriram com o que foi apresentado no início da pesquisa.

Helgeson, Voss e Terpening (2002) destacam que as recusas são baseadas, em grande medida, em construções de atitudes, ao invés de percepções negativas com relação ao instrumento de pesquisa. Além disso, os participantes que se sentem pressionados pelo tempo são susceptíveis de perceber qualquer tempo gasto em pesquisa como um sacrifício.

Considerou-se, no entanto, para a pesquisa SAPIENS-Brasil, que o diálogo com os professores colaboradores, possibilitaria a contextualização dos resultados encontrados, bem como esclareceria detalhes da aplicação do instrumento de pesquisa, no caso, o questionário. A proposta de encontro com esses docentes seria um momento para troca de experiências e também de formação dos professores, uma vez que seria realizado em conjunto com o evento Encontro de História e Filosofia da Biologia em Ribeirão Preto - SP, de modo a garantir a participação dos docentes em palestras e grupos de discussões com profissionais de várias instituições e regiões do país.

Essa aproximação teve o intuito de possibilitar o contato direto com os professores e inseri-los na condição de sujeitos ativos e participativos da pesquisa, e não somente como 
executores para a obtenção de dados para as pesquisas. A viabilização desse encontro com professores de diferentes regiões do país contribuiria também para ampliar o processo de formação e atualização de seus conhecimentos, visto que eles raramente têm a oportunidade de se ausentar do trabalho e realizar cursos de formação, devido à carga horária didática excessiva.

Considerando a advertência de Telles (2002) sobre o problema da falta de retorno dos pesquisadores às escolas pesquisadas, acreditou-se que estabelecer maior proximidade com os docentes e consequentemente com a escola seria uma contrapartida inicial da universidade. Além disso, como citado anteriormente, as 78 escolas que participaram da pesquisa SAPIENS-Brasil receberam os dados de sua escola comparados com os dados gerais brasileiros, de modo que a universidade não se limitou à coleta, mas apresentou o resultado de cada instituição. Helgeson, Voss e Terpening (2002) sugerem que esse retorno pode fomentar a curiosidade dos sujeitos, que deverá se manifestar no seu maior envolvimento na pesquisa.

Ademais, a partir de uma perspectiva de pesquisa de cunho colaborativo, é possível obter resultados sobre as diferentes dimensões das escolas brasileiras, contextualizando os resultados com a cultura escolar e assumindo uma postura crítica referente ao instrumento de avaliação. Desse modo, a discussão dos resultados das pesquisas avaliativas no âmbito colaborativo pode dar suporte para que professores e pesquisadores se posicionem criticamente com relação às medidas de políticas públicas que eventualmente sejam tomadas.

\section{Parceria universidade-escola: desafios e possibilidades no âmbito da pesquisa}

No primeiro contato com as escolas, destacou-se a falta de confiança de alguns gestores na parceria universidade-escola, o que, por consequência, fez com que não concordassem com a participação de seus alunos e professores no projeto, pelas razões apresentadas anteriormente; houve escolas que não confiaram nos retornos oferecidos, $a$ priori, mas, mesmo assim, optaram por aderir à proposta.

Notou-se o estranhamento de gestores e docentes ao receberem, pela primeira vez, a oportunidade de interagirem com uma universidade da região Sudeste e com escolas de várias regiões, além da possibilidade de deslocamento do professor para participar de um evento científico nacional e dialogar com professores de outras regiões que também participaram da pesquisa. 
A terceira situação que merece relevância foi a dificuldade de colaboração entre a universidade e a escola pela evidente incompatibilidade de metas e objetivos de ambas. Algumas escolas brasileiras optaram por não se envolver com a pesquisa justamente porque previa a participação do professor em um evento científico, o que causaria transtornos à dinâmica escolar, pois o docente precisaria se ausentar das suas atribuições diárias em sala de aula e seria, portanto, penalizado com faltas, já que o evento aconteceria em dias letivos. Assim, enquanto a universidade apresentava como metas aproximar a academia da escola e favorecer o diálogo entre docentes e pesquisadores sobre as opiniões de estudantes brasileiros, a escola, por outro lado, destacava a meta de cumprir com o calendário letivo e evitar que as aulas e a continuidade de conteúdo escolar fossem interrompidas.

O envolvimento do professor da Educação Básica em eventos científicos que discutem resultados de pesquisas desenvolvidas no âmbito escolar esbarra também nas condições de trabalho que lhe são oferecidas, pois, em decorrência do excesso de exigências e de horas em sala de aula, o trabalho desse profissional passou a ser compreendido como o de executor de aulas, sem tempo nem espaço em seu cotidiano para o envolvimento com pesquisas.

A Tabela 1 mostra as cidades, regiões e o número de professores que participaram do evento científico na Universidade de São Paulo, campus de Ribeirão Preto, e que colaboraram com pesquisas integradas ao projeto SAPIENS-Brasil.

Tabela 1 - Distribuição dos professores, por região

\begin{tabular}{|c|c|c|}
\hline \multicolumn{2}{|c|}{ Regiões/Cidades } & $\begin{array}{l}\text { Número de } \\
\text { Professores }\end{array}$ \\
\hline \multirow{6}{*}{ Norte } & Manicoré (AM) & 1 \\
\hline & Manaus (AM) & 1 \\
\hline & Amapá (AP) & 1 \\
\hline & Cacoal (RO) & 2 \\
\hline & Porto Velho (RO) & 1 \\
\hline & Tocantins (TO) & 1 \\
\hline \multirow{7}{*}{ Nordeste } & Feira de Santana (BA) & 1 \\
\hline & Rio Grande do Norte (NA) & 1 \\
\hline & Aurora do Ceará (CE) & 1 \\
\hline & Fortaleza $(\mathrm{CE})$ & 1 \\
\hline & Viçosa do Ceará (CE) & 1 \\
\hline & Maceió (AL) & 2 \\
\hline & São Luís (MA) & 1 \\
\hline Centro-Oeste & Brasília (DF) & 1 \\
\hline Sudeste & $\begin{array}{l}\text { Cachoeiro de Itapemirim } \\
\text { (ES) }\end{array}$ & 1 \\
\hline Sul & Rosário do Sul (RS) & 1 \\
\hline Total & & 18 \\
\hline
\end{tabular}

Fonte: Elaboração própria. 
Em comparação com o número total (78) de professores que colaboraram com a aplicação do questionário (e que constituiu o corpus de uma das pesquisas integrantes aos projetos ROSE e SAPIENS), o número reduzido de professores (18) que participaram do encontro se justifica pelas limitações orçamentárias do projeto de pesquisa com despesas de deslocamentos terrestres, aéreos e de hospedagem.

Há que se destacar, no entanto, que, apesar das dificuldades apresentadas, a participação dos professores da Escola Básica na condução da pesquisa, dando-lhes voz enquanto parceiros de um processo de investigação das opiniões de seus alunos, e o retorno dos dados coletados para cada escola contribuíram para uma parceria mais efetiva, uma vez que escola, professores e gestores atenderam aos prazos de coletas e de envio dos questionários respondidos conforme as orientações previstas. Durante os contatos telefônicos e o encontro em Ribeirão Preto (SP), os professores manifestaram o sentimento de valorização, pois se sentiram integrantes e habilitados a colaborar com a pesquisa em ensino.

De acordo com Mclaughlin e Black-Hawkins (2004), é possível identificar vários tipos de parcerias entre escola e universidade, entretanto, o ponto crucial para a eficácia dessa proximidade é o cuidado com a natureza dos relacionamentos que são estabelecidos. É necessário construir uma parceria de respeito mútuo, confiança e um senso de valorização para todos os parceiros, de modo que as relações se desenvolvam como a própria parceria evolui.

\section{Considerações Finais}

O desenvolvimento de projetos de pesquisa de cunho quantitativo, e em nível nacional, revelou-se como um processo permeado por vários desafios, mas, sobretudo, perceberam-se as reais possibilidades de se realizar coletas de dados com representatividade nacional. Desde as experiências reportadas por Santos-Gouw (2013) e Mota (2013), a principal dificuldade de uma investigação em grande escala é estabelecer estratégias para aumentar a proporção de respostas das escolas e devoluções dos questionários através dos serviços de correio. De outra parte, entre os desafios também se destacaram as parcerias de confiança entre universidade e escola, que nem sempre se revelam como uma negociação harmoniosa. As experiências com as pesquisas integradas aos projetos SAPIENS e ROSE apontam para a necessidade de se realizar a pesquisa de forma integrada e colaborativa, na qual todos os sujeitos atuam como 
pesquisadores e formadores, pois, se assim não o fizerem, corre-se o risco de reforçar as tensões existentes entre a universidade e a escola básica.

Garantir a alta proporção de respostas das escolas não depende apenas do empenho dos pesquisadores com o envio dos questionários acompanhados de instruções de aplicação. Também se revelaram elementos importantes a participação, o envolvimento e, principalmente, o empenho na realização de um projeto em conjunto entre a universidade e a escola, pelos professores e gestores do ensino básico. Assim, para superar a impressão de que a escola seria utilizada apenas como campo de coleta de dados, seguida do abandono pelos pesquisadores, foram estabelecidos retornos concretos para as escolas e para os professores colaboradores, a fim de fazer com que ocupassem o lugar de formadores nos campos das pesquisas educacionais.

Em suma, no que se refere às questões metodológicas, o presente trabalho procurou demonstrar que é possível desenvolver projetos de pesquisa com amostras nacionais. E, para minimizar o problema da baixa proporção de resposta, alguns critérios adotados são sugeridos:

1) Constituição e planejamento do projeto de pesquisa por uma equipe de pesquisadores;

2) Contatos telefônicos prévios e ao longo das coletas de dados, a fim de garantir a proximidade com os parceiros do ensino básico e o cumprimento dos prazos;

3) Atenção e cuidado com as características e o formato do questionário (evitando-se instrumentos extensos), bem como com os envelopes, que devem ser selados para retorno dos questionários respondidos e conter: Termo de Consentimento Livre e Esclarecido e orientações para os professores acerca da aplicação do instrumento;

4) Estabelecimento e apresentação clara dos objetivos do projeto de pesquisa para os parceiros, bem como esclarecimento prévio das contribuições da participação da escola e do envolvimento dos professores com o projeto e, principalmente, cumprir com os acordos estabelecidos, para estabelecer uma relação de confiança.

\section{REFERÊNCIAS}

BIZZO, N.; PELLEGRINI, G. Os jovens e a ciência. Curitiba: CRV, 2013. 154p. 
CRESWELL, J. W. Research Design: qualitative \& quantitative approaches. Thousand Oaks; London; New Delhi: SAGE Publications, 1994.

FASSINGER, R.; MORROW, S. L. Toward Best Practices in Quantitative, Qualitative, and Mixed-Method Research: A Social Justice Perspective. Journal for Social Action in Counseling and Psychology, v. 5, n. 2, p. 69-83, 2013.

FLICK. U. Qualidade na pesquisa qualitativa. Tradução de Roberto Dirceu da Silva. Porto Alegre: Artmed, 2009. 196p.

GATTI, B. A. Estudos quantitativos em educação. Educação e Pesquisa, v. 30, n. 1, p. 11 30, 2004.

GOUVEIA, V. V.; GÜNTHER, H. Taxa de resposta em levantamento de dados pelo correio: o efeito de quatro variáveis. Psicologia: Teoria e Pesquisa, v. 11, n. 2, p. 163-168, 1995.

HELGESON, James G.; VOSS, Kevin E.; TERPENING, Willbann D. Determinants of mail-survey response: Survey design factors and respondent factors. Psychology \& Marketing, v. 19, n. 3, p. 303-328, 2002.

MARCONI, M. A.; LAKATOS, E. M. Fundamentos de metodologia científica. 6. ed. São Paulo: Atlas, 2007, 315 p.

MCLAUGHLIN C.; BLACK-HAWKINS, K. A Schools-University Research Partnership: understandings, models and complexities. Journal of In-Service Education, v. 30, n. 2, p. 265-284, 2004.

MILLER, P. M.; HAFNER, M. M. Moving Toward Dialogical Collaboration: A Critical Examination of a University - School - Community Partnership. Educational Administration Quarterly, v. 44, n. 1, p. 66-110, 2008.

MINAYO, M. C. S. O desafio do conhecimento: pesquisa qualitativa em saúde. 8. ed. São Paulo: Hucitec, 2004. 264p.

MINAYO, M. C. S.; SANCHES, O. Quantitativo-Qualitativo: Oposição ou Complementaridade? Cadernos de Saúde Pública, Rio de Janeiro, v. 9, n. 3, p. 239-262, 1993.

MOTA, H. S. Evolução biológica e religião: atitudes de jovens estudantes brasileiros. 2013. 275 f. Tese (Doutorado em Educação) - Faculdade de Educação, Universidade de São Paulo, São Paulo, 2013.

OLIVEIRA, G. S. Estudantes e a evolução biológica: conhecimento e aceitação no Brasil e Itália. 2015. 317 f. Tese (Doutorado) - Faculdade de Educação, Universidade de São Paulo, São Paulo, 2015.

SAMPIERI, R. H; COLLADO, C. H.; LUCIO, P. B. Metodologia de pesquisa. 3. ed. Tradução de Fátima Conceição Murad, Melissa Kassner, Sheila Clara Dystyler Ladeira. São Paulo: McGraw-Hill, 2006. 583p. 
SANTOS-GOUW, A. M. As opiniões, interesses e atitudes dos jovens brasileiros frente à ciência: uma avaliação em âmbito nacional. 2013. 242 f. Tese (Doutorado em Educação) Faculdade de Educação, Universidade de São Paulo, São Paulo, 2013.

SCHREINER, C.; SJØBERG, S. Sowing the seeds of ROSE. Acta Didactica 4/2004. 2004, 120 p.

TELLES, J. A. "É pesquisa, é? Ah, não quero, não, bem!” Sobre pesquisa acadêmica e sua relação com a prática do professor de línguas. Linguagem \& Ensino, v. 5, n. 2, p. 91-116, 2002.

\section{SOBRE OS AUTORES:}

\section{Graciela da Silva Oliveira}

Doutora em Educação pela Universidade de São Paulo (USP). Docente do Instituto de Biociências, da Universidade Federal de Mato Grosso (UFMT). Campus de Cuiabá - MT. Email: graciela.ufmt@gmail.com

iD http://orcid.org/0000-0003-0609-8558

\section{Ana Paula Zampieri Silva de Pietri}

Doutora em Biologia pela Universidade Estadual Paulista (UNESP). Gestora de Projetos Pedagógicos em Educação Integral - Associação Cidade Escola Aprendiz (Centro de Referências em Educação Integral). São Paulo - SP. E-mail: anapaulapietri@ aprendiz.org.br

(iD) http://orcid.org

\section{Nelio Bizzo}

Doutor em Educação pela Universidade de São Paulo (USP). Professor Titular da Faculdade de Educação da Universidade de São Paulo e Universidade Federal de São Paulo, Campus Diadema. Coordenador científico do Núcleo de Pesquisa em Educação, Divulgação e Epistemologia da Evolução (EDEVO-Darwin), da Pró-Reitoria de Pesquisa da USP e do Projeto Temático BIOTA/Fapesp-Educação (2018-2023), que articula cinco instituições acadêmicas (USP, UFABC, UNIFESP, USCS e Instituto Butantan). E-mail: bizzo@usp.br (iD) http://orcid.org/0000-0002-7209-2659 